\title{
Physical Characterization of Active Asteroid (6478) Gault
}

\author{
Juan A. Sanchez ${ }^{1}$, Vishnu Reddy ${ }^{2}\left(\mathbb{0}\right.$, Audrey Thirouin $^{3}$ (D), Edward L. Wright ${ }^{4}\left(\mathbb{D}\right.$, Tyler R. Linder ${ }^{5,6}$, Theodore Kareta ${ }^{2}$ (D), and \\ Benjamin Sharkey ${ }^{2}$ \\ ${ }^{1}$ Planetary Science Institute, 1700 East Fort Lowell Road, Tucson, AZ 85719, USA; jsanchez@psi.edu \\ ${ }^{2}$ Lunar and Planetary Laboratory, University of Arizona, 1629 East University Blvd, Tucson, AZ 85721-0092, USA \\ ${ }^{3}$ Lowell Observatory, 1400 West Mars Hill Road, Flagstaff, AZ 86001, USA \\ ${ }^{4}$ Division of Astronomy and Astrophysics, University of California Los Angeles, 430 Portola Plaza, Box 951547, Los Angeles, CA 90095-1547, USA \\ ${ }^{5}$ Astronomical Research Institute, 1015 Cr 1300N, Sullivan, IL 61951, USA \\ ${ }^{6}$ University of North Dakota, Clifford Hall Room 512, 4149 University Avenue Stop 9008, Grand Forks, ND 58202, USA \\ Received 2019 April 18; revised 2019 July 11; accepted 2019 July 12; published 2019 August 5
}

\begin{abstract}
Main belt asteroid (6478) Gault has been dynamically linked with two overlapping asteroid families: Phocaea, dominated by S-type asteroids, and Tamara, dominated by low-albedo C-types. This object has recently become an interesting case for study after images obtained in late 2018 revealed that it was active and displaying a comet-like tail. Previous authors have proposed that the most likely scenarios to explain the observed activity on Gault were rotational excitation or merger of near-contact binaries. Here we use new photometric and spectroscopic data of Gault to determine its physical and compositional properties. Lightcurves derived from the photometric data showed little variation over three nights of observations, which prevented us from determining the rotation period of the asteroid. Using WISE observations of Gault and the near-Earth Asteroid Thermal Model (NEATM) we determined that this asteroid has a diameter $<6 \mathrm{~km}$. Near-infrared spectroscopic data obtained with the Infrared Telescope Facility showed a spectrum similar to that of S-complex asteroids, and a surface composition consistent with $\mathrm{H}$ chondrite meteorites. These results favor a compositional affinity between Gault and asteroid (25) Phocaea, and rules out a compositional link with the Tamara family. From the spectroscopic data we found no evidence of fresh material that could have been exposed during the outburst episodes.
\end{abstract}

Key words: minor planets, asteroids: general - techniques: spectroscopic

\section{Introduction}

Asteroid (6478) Gault is a $<10 \mathrm{~km}$ sized object located in the inner part of the main belt $(a \sim 2.3 \mathrm{au})$ in the Phocaea family. This asteroid family is composed of nearly 2000 members and it is dominated by S-type asteroids (Carruba 2009; Nesvorný et al. 2015). Observations obtained by the Zwicky Transient Facility (ZTF) survey showed that Gault experienced two brightening events, one on 2018 October $18 \pm 5$ days and the other on 2018 December $24 \pm 1$ days (Ye et al. 2019). Images obtained by the Asteroid Terrestrial-impact Last Alert System (ATLAS) on 2018 December 8 revealed that Gault became active, displaying a $30^{\prime \prime}$-long tail at a position angle $(\mathrm{PA})=290^{\circ}$. On 2019 January 5, new images of Gault obtained by ATLAS showed that the tail was measuring 135"-long (Smith et al. 2019), and later that month a second dust tail was detected (Jehin et al. 2019). This discovery prompted Chandler et al. (2019) to look for signs of activity among NOAO archived images, which revealed that Gault has been active for at least six years, as seen in images taken in 2013 when a pronounced tail was already present.

Based on photometric measurements obtained by the ZTF survey, Ye et al. (2019) found that the dust ejecta was dominated by grains of up to $10 \mu \mathrm{m}$ in size that are ejected at low velocities $\left(<1 \mathrm{~m} \mathrm{~s}^{-1}\right)$, which is indicative of non-sublimation-driven ejections. They suggested that the most likely scenarios to explain the activity of this asteroid were rotational excitation or merger of near-contact binaries.

Moreno et al. (2019) obtained photometric data of Gault on 2019 January 13, 14, and 15 using various telescopes around the world in order to determine its rotation period. However, the lightcurves showed no significant variation over time. Their analysis of the dust properties yielded results consistent with those of Ye et al. (2019).

Kleyna et al. (2019) carried out ground-based observations of Gault from 2019 February 8 to 18 . From their photometric data they determined that Gault has a rotation period of $\sim 2 \mathrm{hr}$. They noticed that this value is close to the critical break-up limit of a rubble pile at $\sim 2.2 \mathrm{hr}$ per rotation (e.g., Pravec \& Harris 2000), suggesting that the dust emission was caused by disruption or landslides resulting from a YarkovskyO'Keefe-Radzievskii-Paddack (YORP)-induced rotational disturbance.

Although Gault is dynamically linked with the Phocaea family, its spectral and compositional affinity with this family have not been confirmed. Broadband colors of Gault showed that this object is more similar to C-type asteroids than S-types (Jewitt et al. 2019; Ye et al. 2019). Furthermore, Kleyna et al. (2019) found that Gault is also dynamically linked with the low-albedo Tamara family, a recently discovered asteroid family residing in the Phocaea region (Novaković et al. 2017).

In this study we present new photometric data from the Infrared Telescope Facility (IRTF) and the Cerro Tololo InterAmerican Observatory (CTIO) to verify the $2 \mathrm{hr}$ rotation period reported by Kleyna et al. (2019). We also constrain the diameter of Gault using data from the WISE mission. In addition, near-infrared (NIR) spectra of Gault obtained with the IRTF are used to determine its taxonomic type, composition, and possible meteorite analogs. We also use the spectroscopic data to search for signs of fresh material that could have been excavated when the asteroid became active. 


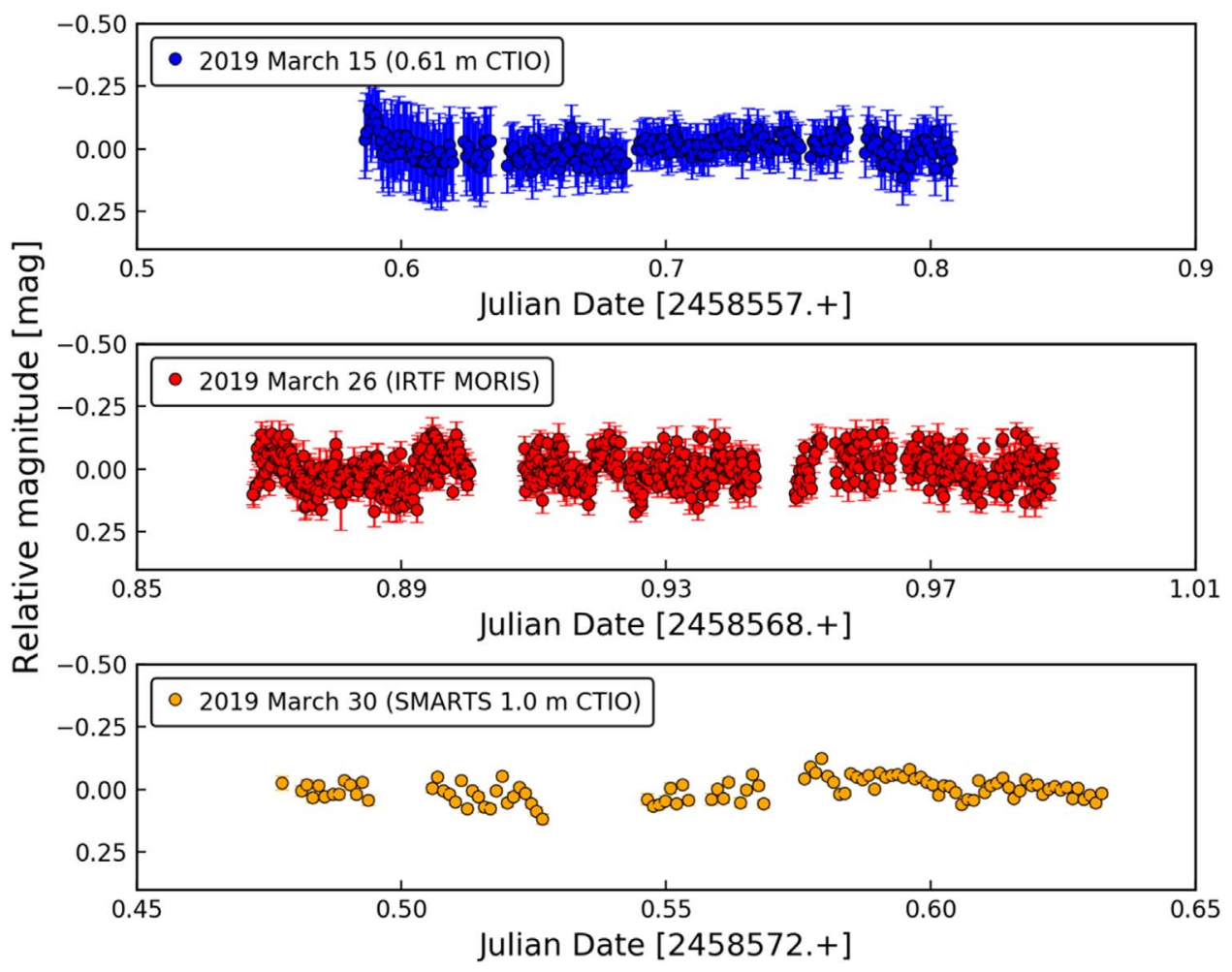

Figure 1. Lightcurves of (6478) Gault presented in chronological order with data obtained on 2019 March 15 with the $0.61 \mathrm{~m} f / 6.8$ at the CTIO in blue, IRTF MORIS data obtained on March 26 in red, and data from March 30 obtained with the SMARTS $1.0 \mathrm{~m}$ telescope at CTIO in orange. We find no obvious rotational period as suggested by Kleyna et al. (2019).

\section{Physical Characterization}

\subsection{Photometric Observations}

Gault was observed for rotational lightcurve study on three occasions over two weeks. Our first data set was obtained through a clear filter on a $0.61 \mathrm{~m} f / 6.8$ telescope and an Apogee F6 camera with a pixel scale of 1"! 4/pixel and a field of view of $24^{\prime}$ square located at the CTIO facility on 2019 March 15, an exposure time of $60 \mathrm{~s}$ was used. On March 26, we used the MIT Optical Rapid Imaging System (MORIS) instrument on NASA IRTF with a pixel scale of 0 "! $11 /$ pixel and a field of view of $1^{\prime}$ square. MORIS data were taken using an LPR600 filter with an exposure time of $8 \mathrm{~s}$. On March 30, we collected unfiltered observations with the SMARTS $1.0 \mathrm{~m}$ telescope at CTIO using an Apogee F42 with a pixel scale of 1"!05 and a field of view of $9^{\prime}$ square, exposures were limited to $90 \mathrm{~s}$. The CTIO data sets were both calibrated and reduced with the Canopus software, whereas the MORIS data set was analyzed with the techniques described in Thirouin et al. (2010).

Two tails were seen in the images obtained at the CTIO, the first was measured to be $\sim 167^{\prime \prime}$-long and the second was $5^{\prime \prime}$-long. In a stack of the images taken at CTIO, we measured the asteroids point-spread function (PSF) away from the two tails and found it to have an FWHM of 23.05 pixels (3!"2); this is similar to the stars in the image, which have FWHMs of 2.95-3.2 pixels. Thus, while we see no direct evidence for an extended dust coma, if it is present it should have a lower surface brightness than either of the two tails detected.

As we did not measure any extended reflected light coming from Gault brighter than either of the tails, we use the brightness measured for both tails to determine their contribution as a rough estimate of the overall dust contamination of our spectral data. We converted a set of the CTIO images stacked on Gault to polar coordinates and measured the radial intensity profiles along both tails, as well as in a direction away from either tail as mentioned previously. We calculated the increase in brightness by subtracting the away-from-tails profile from the short and long tails. Compared to the brightest pixel of Gault, the short tail is found to be $\sim 6 \%$ as bright and the long tail is found to be $1.5 \%-2.0 \%$ as bright in the region within several arcseconds of the asteroid. Considering the limitations of the methods, we argue that the light observed is dominated by reflected light from the asteroid's surface and that the two tails make up $\sim 8 \%$ of the light detected.

\subsection{Lightcurve Results}

Our three data sets span $\sim 5 \mathrm{hr}, \sim 3 \mathrm{hr}$, and $\sim 4 \mathrm{hr}$, respectively. Cloudy conditions during the first observing run affected the quality of the photometry. All data sets show a flat lightcurve with no obvious variability over the duration of the observing blocks (Figure 1). Our data sets were inspected for periodicities with the Lomb periodogram, and the Phase Dispersion Minimization technique (Lomb 1976; Stellingwerf 1978). First, we tested each data set individually, and in a second step we searched for periodicity using the whole sample. In all cases, no rotational period was favored with a high enough $(>99.9 \%)$ confidence level.

As stated earlier, the lightcurve of Gault was also studied in Moreno et al. (2019) and Kleyna et al. (2019). Our flat lightcurve is consistent with the Moreno et al. (2019) results. Based on our data, we cannot confirm the rotational period of $\sim 2 \mathrm{hr}$ for Gault (assuming a double-peaked lightcurve) reported by Kleyna et al. (2019). These results are compatible with an asteroid observed pole-on, or an object having a 


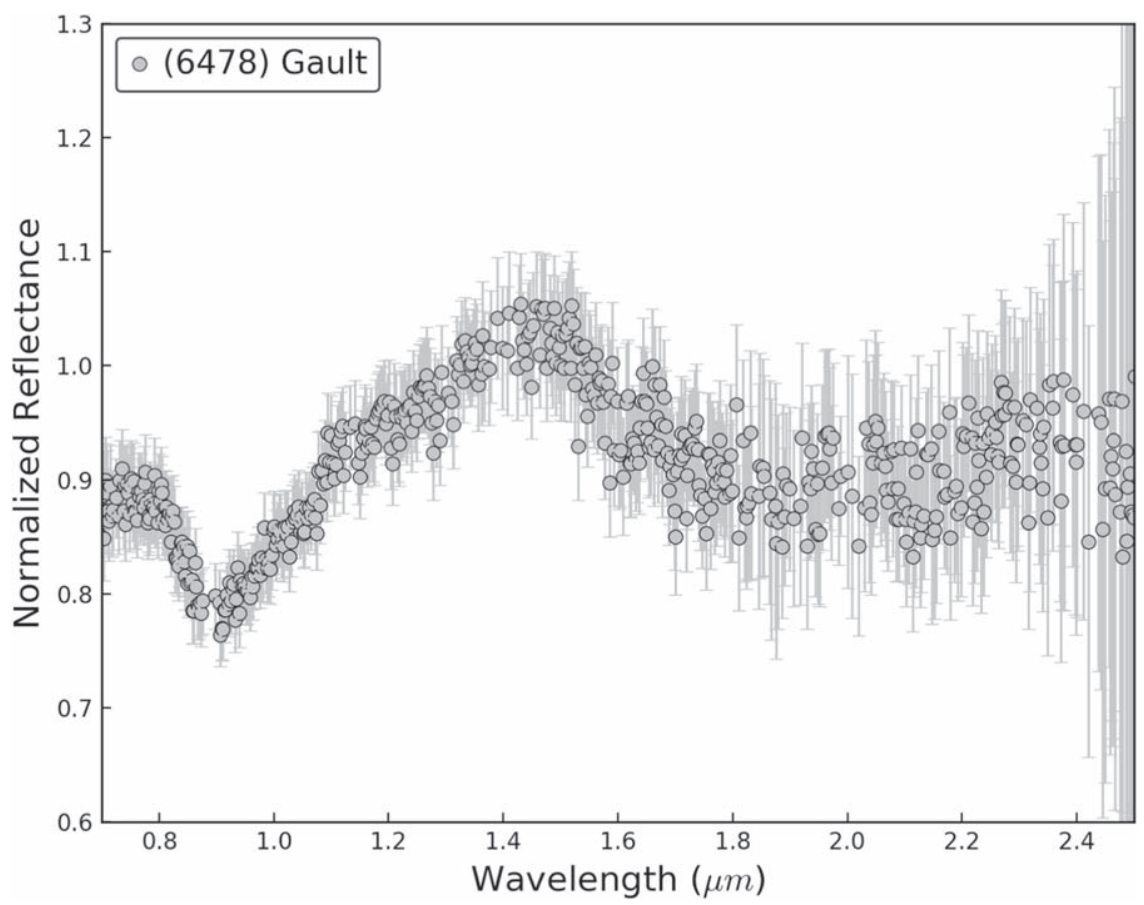

Figure 2. NIR spectrum of (6478) Gault obtained using the SpeX instrument on NASA IRTF. The spectrum exhibits two absorption bands at $\sim 1$ and $2 \mu \mathrm{m}$ due to the presence of olivine and pyroxene. The spectrum is normalized to unity at $1.5 \mu \mathrm{m}$.

spherical shape. As discussed by Moreno et al. (2019), the presence of dust around the asteroid would further complicate the analysis of a lightcurve with a small amplitude.

\subsection{Thermal Modeling}

The only WISE observations of Gault in the Minor Planet Center database are 15 detections centered on 2016 August 25.6, when it was near perihelion and only 1.39 au from the Earth. But a search of the WISE image archive shows 21 frames covering the position of Gault. Clearly the flux was close to the detection limit, so positive noise fluctuations would get detected while negative fluctuations would not. To avoid bias, a PSF fit to the pixels surrounding Gaul on all 21 frames was done, yielding $W 1=$ $15.912 \pm 0.091 \mathrm{mag}$ and $W 2=13.856 \pm 0.033 \mathrm{mag}$. The heliocentric distance was $r=1.89 \mathrm{au}$ and the phase angle was $\alpha=31^{\circ} .8$. A similar search of the cryogenic phases of the WISE mission showed 16 frames during the three-band cryo phase centered on 2010 August 26.9. PSF fits to the pixels in these frames give $W 1=17.72 \pm 0.32 \mathrm{mag}, W 2=16.11 \pm 0.33 \mathrm{mag}$, and $W 3=9.00 \pm 0.24$ mag. Gault was $\Delta=2.38$ au from Earth, $r=2.57$ au from the Sun, with a phase angle $\alpha=23^{\circ} .1$ during these observations.

A thermal model NEATM (Harris 1998) fit to these WISE data plus an absolute magnitude $(H)$ of $14.4 \pm 0.3$ was performed, but the combination of the low signal-to-noise ratio $(\mathrm{S} / \mathrm{N})$ in the $W 2-W 3$ color and the uncertain contribution of reflected light to the $W 2$ flux required the addition of prior knowledge to stabilize the fit. We ran a Bayesian calculation using a Monte Carlo Markov chain having log normal priors with $p_{\mathrm{IR}} / p_{V}=1.76 \pm 34 \%$ and $\eta=1.2 \pm 29 \%$. The posterior of the fit had a geometric albedo $p_{V}=0.176 \pm 44 \%, p_{\mathrm{IR}} / p_{V}=$ $1.39 \pm 24 \%, \eta=1.36 \pm 18 \%$, and a diameter $D=3.96 \pm$ $22 \% \mathrm{~km}$. A caveat is that the diameter and beaming are tightly correlated in the posterior with $D \propto \eta^{1.2}$, and the prior contributes $39 \%$ of the weight in the $\eta$ value, so the $\eta$ prior still has an important effect on the diameter value. The distribution of $\eta$ seen in Masiero et al. (2014) has a median of 0.9 and a $1 \sigma$ range of $\pm 13 \%$ with only five out of 3080 objects showing $\eta$ greater than the $+1 \sigma$ point $(\eta=1.615)$ in the posterior for Gault. Thus, a diameter greater than the posterior $+2 \sigma$ point of $6.1 \mathrm{~km}$ is unlikely.

\subsection{Spectroscopic Observations}

We carried out observations of Gault on 2019 March 26 UTC with the SpeX instrument (Rayner et al. 2003) on the IRTF. NIR spectra $(0.7-2.5 \mu \mathrm{m})$ of the asteroid, extinction, and solar analog stars were obtained in low-resolution $(R \sim 150)$ prism mode with a 0 ." 8 slit width. All spectra were obtained at the parallactic angle to minimize differential refraction at the shorter wavelength end.

A total of $30200 \mathrm{~s}$ spectra of Gault were acquired when the asteroid was 17.8 visual magnitude, at a phase angle of $14^{\circ}$. A G-type local extinction star was observed before and after the asteroid observations in order to correct the telluric absorption bands. NIR spectra of solar analog star SAO 120107 were also obtained to correct for spectral slope variations that could be introduced by the use of a non-solar local extinction star. Data reduction was performed using the IDL-based software Spextool (Cushing et al. 2004). A detailed description of the data reduction procedure is presented in Sanchez et al. (2013, 2015).

\subsection{Compositional Analysis}

The NIR spectrum of Gault is shown in Figure 2. Two absorption bands at $\sim 1$ and $2 \mu \mathrm{m}$ due to the presence of olivine and pyroxene can be seen. We have performed the taxonomic classification of the asteroid using the online Bus-DeMeo taxonomy calculator (http://smass.mit.edu/busdemeoclass. html), which showed that Gault belongs to the S-complex, specifically to the Sr-type $\left(\mathrm{PC}^{\prime}=0.1636, \mathrm{PC}^{\prime}=0.0679\right)$ 


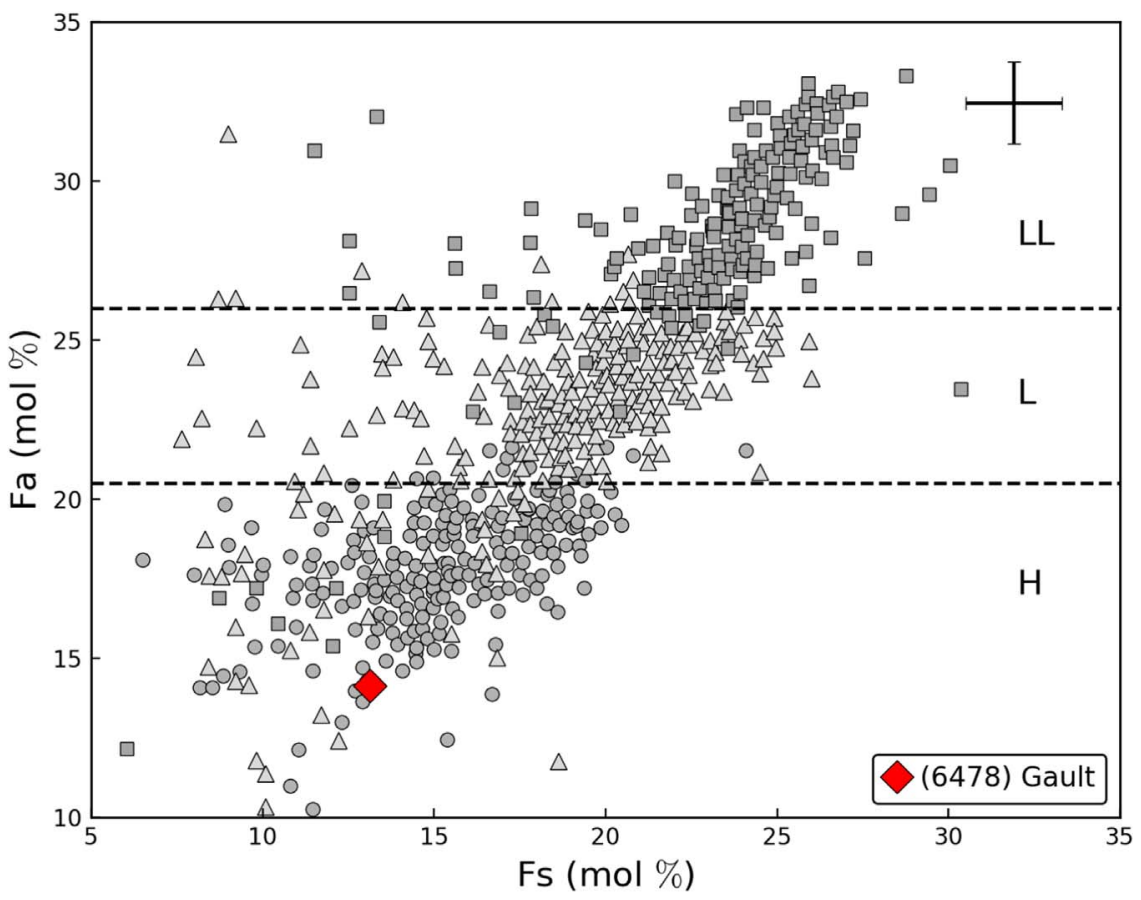

Figure 3. Iron abundance in silicate minerals on Gault represented as mol\% of fayalite (Fa) vs. ferrosilite (Fs). Measured values for LL (squares), L (triangles), and $\mathrm{H}$ (circles) ordinary chondrites from Nakamura et al. (2011) are also included. The error bars in the upper-right corner correspond to the uncertainties derived by Dunn et al. (2010), $1.3 \mathrm{~mol} \%$ for Fa, and $1.4 \mathrm{~mol} \%$ for Fs. Figure adapted from Nakamura et al. (2011).

under this taxonomic system (DeMeo et al. 2009). Sr-types typically exhibit a deeper $2 \mu \mathrm{m}$ band than S-types; however, we noticed that the large scattering of the data in this band is likely affecting the $\mathrm{PC}^{\prime}$ value, which increases as this band becomes deeper, thus it is also possible that the object is an S-type.

Spectral band parameters including band centers, band depths, and band area ratio (BAR), as well as their associated errors, were measured using a Python code following the procedure described in Sanchez et al. (2012). We used the measured Band $I$ center $(0.92 \pm 0.01 \mu \mathrm{m})$ and the equations of Dunn et al. (2010) to determine the olivine and pyroxene chemistry, which are given by the molar contents of fayalite $(\mathrm{Fa})$ and ferrosilite $(\mathrm{Fs})$, respectively. The BAR can be used to calculate the olivine-pyroxene abundance ratio $(\mathrm{ol} /(\mathrm{ol}+\mathrm{px}))$; however, the scattering of the data in the $2 \mu \mathrm{m}$ band could make this parameter unreliable, therefore we decided not to use it. The olivine and pyroxene chemistries of Gault were found to be $\mathrm{Fa}_{14.1 \pm 1.3}$ and $\mathrm{Fs}_{13.1 \pm 1.4}$, respectively. Figure 3 shows the molar content of Fa versus Fs for Gault and measured values for LL, L, and $\mathrm{H}$ ordinary chondrites from Nakamura et al. (2011). Within the uncertainties, these values fall in the range of those found for $\mathrm{H}$ chondrites by Dunn et al. (2010). This result rules out a compositional affinity between Gault and the Tamara family, whose members are thought to be low-albedo $(<0.1)$ C-types, and would favor a link between Gault and asteroid (25) Phocaea, whose composition has been found to be consistent with ordinary chondrites. Because S-type asteroids are composed of anhydrous silicates, a volatile-driven activity for Gault seems unlikely.

\subsection{Looking for Fresh Excavated Material}

Different mechanisms have been proposed to explain dust ejection from asteroids, including volatile sublimation, rotational mass loss, impacts, and thermal disintegration, among others (Jewitt et al. 2015). As stated earlier, in the case of Gault, the merger of near-contact binaries or rotational breakup are thought to be the physical mechanisms behind the activity of the asteroid (Kleyna et al. 2019; Ye et al. 2019). Regardless of what led to the activation of Gault, it would be reasonable to think that landslides and dust ejection could have excavated fresh material from beneath the surface of the asteroid. The NIR spectrum of this fresh material would exhibit deeper absorption bands and a relatively flat spectral slope compared to that of the weathered material. Ideally, the best way to look for signs of fresh exposed material would be to compare spectra obtained before and after the asteroid became active; unfortunately, no pre-outburst spectral data of Gault exist. However, this limitation can be overcome by comparing the spectrum of Gault with those of Q-, Sq-, and S-type asteroids, as these taxonomic types are thought to represent a weathering gradient, with Q-types having relatively fresh surfaces, and Sq- and S-types having more space-weathered surfaces (e.g., Binzel et al. 2010, 2019; Reddy et al. 2018). Figure 4 shows the spectrum of Gault and the mean spectrum of a Q-, Sq-, and an S-type asteroid from DeMeo et al. (2009). We found that the Band $I$ depth of Gault $(14.3 \pm 0.5 \%)$ is much closer to that of the S-type $(13.0 \pm 0.2 \%)$ than the Sq$(17.5 \pm 0.3 \%)$ and Q-type $(23.8 \pm 0.1 \%)$. We have also used the principal components $\mathrm{PC}^{\prime}$ and $\mathrm{PC}^{\prime}$ obtained in the previous section to calculate the Space Weathering Parameter $\Delta \eta$ (Binzel et al. 2010). This parameter is given by the scalar magnitude of the space weathering vector defined in the principal component space of the Bus-DeMeo taxonomy (Binzel et al. 2010), and can be used to estimate the degree of space weathering experienced by an asteroid.

For Gault, we determined a value $\Delta \eta=0.608$, which is consistent with an extensively weathered surface. These results suggest three possible scenarios: (1) no fresh material was 


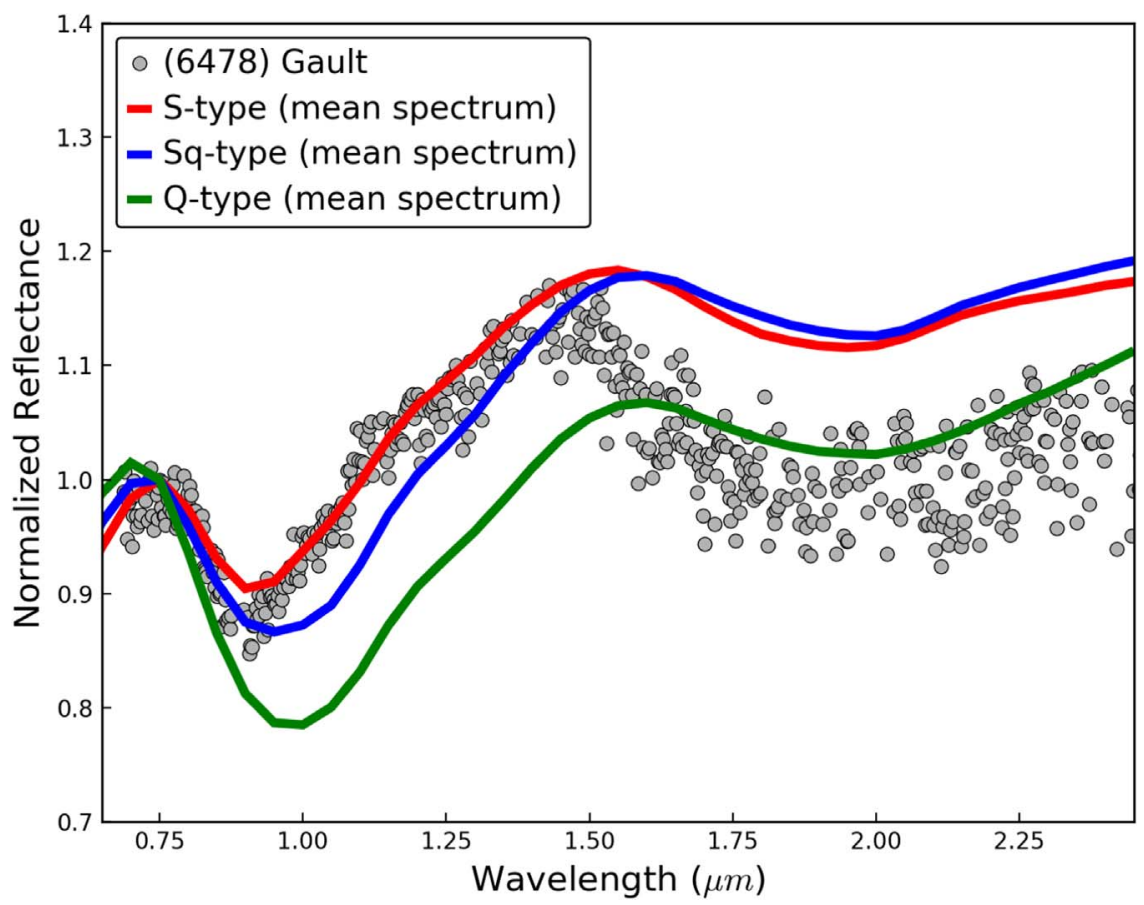

Figure 4. Comparison between the NIR spectra of (6478) Gault and the mean spectra of Q-, Sq-, and S-types from DeMeo et al. (2009). All spectra are normalized to unity at $\sim 0.75 \mu \mathrm{m}$.

excavated; (2) fresh material was excavated but we cannot detect it; (3) our current models for asteroid space weathering are incomplete. In the first scenario, the surface of Gault would have reached a state of saturated space weathering. According to Binzel et al. (2019), under this scenario surface grains can become uniformly weathered after multiple re-arrangements events followed by extended periods of exposure to the space environment, hence causing saturation by space weathering. Thus, when a new re-arrangement of the regolith occurs during a resurfacing event, no fresh material will be exposed. In the second scenario, it is possible that fresh material was excavated in localized regions too small to be detected. Moreno et al. (2019) estimated that the total mass ejected during the 2018 and 2019 events was equivalent to a spherical volume of $\sim 10 \mathrm{~m}$ radius. Because the NIR spectrum obtained with the IRTF is a disk-integrated spectrum, the contribution of such a small region to the overall spectral features would be negligible. In addition, we cannot rule out the possibility that the exposed areas were covered by the re-accumulation of a mixture of fresh and weathered debris. This mechanism has been proposed to explain the lack of spectral variations seen on the site of fission of primary asteroids in asteroid pairs (Polishook et al. 2014). Within the second scenario we also have to consider the possibility that dust could be temporarily masking the spectral signature of the fresh material. However, as discussed in Section 2.1, no evidence for an extended coma was found and it is not clear if the contribution from the tails would be enough for this to happen. Finally, regarding the third scenario, it is also possible that the tools that we are employing to quantify space weathering on asteroids cannot be generalized to all objects, even if they belong to the same taxonomic type. This idea seems to be supported from spacecraft observations of (433) Eros and (243) Ida (both S-types) that show different space weathering trends on these asteroids (Gaffey 2010).

\section{Summary}

We have obtained photometric and NIR spectroscopic data of active asteroid (6478) Gault in an effort to independently confirm previous results and to determine for the first time the composition of this asteroid. Our results can be summarized as follows.

1. From the lightcurves we were unable to confirm the $\sim 2 \mathrm{hr}$ rotation period determined by Kleyna et al. (2019). Our results are consistent with those of Moreno et al. (2019).

2. A thermal model fit to WISE data yielded values of $p_{V}=0.176 \pm 44 \%$, and $D=3.96 \pm 22 \% \mathrm{~km}$ for the geometric albedo and diameter, respectively.

3. The olivine and pyroxene chemistries of Gault were found to be consistent with those of H-type ordinary chondrites, suggesting a compositional affinity with (25) Phocaea.

4. We found no sign of fresh material that could have been exposed during the outburst episodes. This can be seen in the overall shape of the spectrum and the intensity of the $1 \mu \mathrm{m}$ band of Gault, which are more similar to those of an S-type than Sq- and Q-types.

This research work was supported by NASA Near-Earth Object Observations Grant NNX17AJ19G (PI: V. Reddy). We thank the IRTF TAC for awarding time to this project, and to the IRTF TOs and MKSS staff for their support. The authors wish to recognize and acknowledge the very significant cultural role and reverence that the summit of Maunakea has always had within the indigenous Hawaiian community. We are most fortunate to have the opportunity to conduct observations from this mountain. A.T. is partially supported by Lowell Observatory funds. Taxonomic type results presented in this work were determined, in whole or in part, using a Bus-DeMeo Taxonomy 
Classification Web tool by Stephen M. Slivan, developed at MIT with the support of National Science Foundation Grant 0506716 and NASA Grant NAG5-12355. We thank the anonymous referee for the thorough review and comments that helped improve the manuscript.

\section{ORCID iDs}

Vishnu Reddy (iD https://orcid.org/0000-0002-7743-3491

Audrey Thirouin (i) https://orcid.org/0000-0002-1506-4248

Edward L. Wright (10) https://orcid.org/0000-0001-5058-1593

Theodore Kareta (i) https://orcid.org/0000-0003-1008-7499

\section{References}

Binzel, R. P., DeMeo, F. E., Turtelboom, E. V., et al. 2019, Icar, 324, 41 Binzel, R. P., Morbidelli, A., Merouane, S., et al. 2010, Natur, 463, 331 Carruba, V. 2009, MNRAS, 398, 1512

Chandler, C. O., Kueny, J., Gustafsson, A., et al. 2019, ApJL, 877, L12 Cushing, M. C., Vacca, W. D., \& Rayner, J. T. 2004, PASP, 116, 362

DeMeo, F. E., Binzel, R. P., Slivan, S. M., et al. 2009, Icar, 202, 160

Dunn, T. L., McCoy, T. J., sunshine, J. M., et al. 2010, Icar, 208, 789

Gaffey, M. J. 2010, Icar, 209, 564
Harris, A. W. 1998, Icar, 131, 291

Jehin, E., Ferrais, M., Moulane, Y., et al. 2019, CBET, 4606, 1

Jewitt, D., Hsieh, H., \& Agarwal, J. 2015, in Asteroids IV, ed. P. Michel,

F. E. DeMeo, \& W. F. Bottke (Tucson, AZ: Univ. Arizona Press), 221

Jewitt, D., Kim, Y., Luu, J., et al. 2019, ApJL, 876, L19

Kleyna, J. T., Hainaut, O. R., Meech, K. J., et al. 2019, ApJL, 874, L20

Lomb, N. R. 1976, Ap\&SS, 39, 447

Masiero, J. R., Grav, T., Mainzer, A. K., et al. 2014, ApJ, 791, 121

Moreno, F., Jehin, E., Licandro, J., et al. 2019, A\&A, 624, L14

Nakamura, T., Noguchi, T., Tanaka, M., et al. 2011, Sci, 333, 1113

Nesvorný, D., Brož, M., \& Carruba, V. 2015, Asteroids IV, 297

Novaković, B., Tsirvoulis, G., Granvik, M., \& Todović, A. 2017, AJ, 153 , 266

Polishook, D., Moskovitz, N., DeMeo, F. E., \& Binzel, R. P. 2014, Icar, 243, 222

Pravec, P., \& Harris, A. W. 2000, Icar, 148, 12

Rayner, J. T., Toomey, D. W., Onaka, P. M., et al. 2003, PASP, 115, 362

Reddy, V., Sanchez, J. A., Furfaro, R., et al. 2018, AJ, 155, 140

Sanchez, J. A., Michelsen, R., Reddy, V., \& Nathues, A. 2013, Icar, 225, 131

Sanchez, J. A., Reddy, V., Dykhuis, M., et al. 2015, ApJ, 808, 93

Sanchez, J. A., Reddy, V., Nathues, A., et al. 2012, Icar, 220, 36

Smith, K. W., Denneau, L., Vincent, J. B., et al. 2019, CBET, 4594, 1

Stellingwerf, R. F. 1978, ApJ, 224, 953

Thirouin, A., Ortiz, J. L., Duffard, R., et al. 2010, A\&A, 522, A93

Ye, Q., Kelley, M. S. P., Bodewits, D., et al. 2019, ApJL, 874, L16 